\title{
Research of electrical power processes for optimum modeling and design of special electric drives
}

\author{
V.Yu. Karandey \\ Kuban State Technological University \\ Krasnodar, Russian Federation \\ epp_kvy@mail.ru \\ O.B. Popova \\ Kuban State Technological University \\ Krasnodar, Russian Federation \\ popova_ob@mail.ru
}

\author{
B.K. Popov \\ Kuban State Technological University \\ Krasnodar, Russian Federation \\ pbk47@mail.ru \\ V.L. Afanasyev \\ Kuban State Technological University \\ Krasnodar, Russian Federation \\ buguvix@mail.ru
}

\begin{abstract}
In the article the research of electrical power processes is made for optimum modeling and design of special electric drives with asynchronous motors. For creation of various types of electric drives with optimum mass dimensional and power indicators, it is necessary to define precisely values of electric currents windings, the electromotive forces and the corresponding flux linkage in the stator and a rotor of components of the research devices. The main difficulty in the research and determination of parameters is continuous change of these sizes in a consequence of change of electric parameters of the feeding system of tension, the spatial provision of mobile parts of the drive relatively motionless parts and features of the technological process. It leads to change of interposition of axes of the field of the stator and a rotor, phase angles of shift of electric currents and the electromotive forces of components of special electric drives. The research of electrical power processes with use of the methods based on electromechanical transformation of energy allows determining required parameters quite precisely. Determination of value of the savedup energy will allow one to model and design quite precisely without the corresponding experimental adjustment special electric drives. And this approach allows one to determine power parameters at any moment that gives the chance to define instant values of required parameters, including instant values of force and the moment on a shaft of the electric drive. Exact and correct definition of power ratios in the research electric drives will allow one not only to carry out optimum design, but also to create optimum control systems of electric drives taking into account technological features of concrete production.
\end{abstract}

Keywords-special electric drive, the operated asynchronous cascade electric drive, energy transformation, mathematical modelling, the electromagnetic field, electromagnetic system.

\section{INTRODUCTION}

The research of electrical power processes for optimum modeling and design of special electric drives with asynchronous engines is conducted. Special electric drives are quite widely used in modern technological processes and productions [1-3]. By the example of special electric drives with asynchronous engines, which designs are offered and developed by the authors, the system of control examples has been created. Known design of a component of the special electric drive has been chosen as a basis [4]

Mathematical models of the research object have been developed for the existing design of the special electric drive and its components. The models received mathematically have allowed one to define the instant distribution of induction in a gap created by currents of stator windings, the instant distribution of induction in a gap created by the induced currents of a rotor winding, corners between currents and tension in stator and rotor windings and solid angles between axes of fields of stator and rotor windings.

The received expressions are the main ones during not only the modeling and design of special electric drives, but also during creation of exact and optimum control systems of the studied devices [5-10]. For this purpose it is necessary to know the value of the electromagnetic energy which is saved up by the device in the considered time point [11-14]. The most expedient approach when determining electromagnetic energy is the method based on electromechanical transformation of energy. In special electric drives, parameters of instant effort and the moment on a shaft of the device [15] will allow one to define knowledge of electrical power processes quite precisely that is now quite a difficult task without the corresponding experimental adjustment [16-17].

We will define a stream on polar division $\tau$ of a rotor:

$$
\begin{gathered}
\Phi=l_{m} \int_{0}^{\tau} B_{c}(x) d x=-l_{m} \int_{0}^{\tau} B_{\delta m c} \cos \left(\omega^{\prime} t+\frac{\pi}{\tau} x\right) d x= \\
=-\frac{l_{m} \tau}{\pi} B_{\delta m c}\left[\sin \left(\omega^{\prime} t+\pi\right)-\sin \left(\omega^{\prime} t\right)\right],
\end{gathered}
$$

where $\quad \tau$ - polar division of a rotor;

$\Phi$ - magnetic flux;

$l_{m}$ - length of a magnetic part of a component of the electric drive;

$B_{c}$ - induction of the stator; 
$B_{\delta m c}$ - induction amplitude at the time of a maximum of induction of the stator on the first tooth; $\omega^{\prime}$ - angular frequency of field rotation of a rotor. $x$ - the coordinate along a rotor circle lectured clockwise.

Considering that phases in the stator alternate from left to right, and phases in the rotor - from right to left, the formula (1) will take a form:

$$
\begin{gathered}
\Phi_{n}=-\frac{l_{m} \tau}{\pi} B_{\delta m c}\left[\sin \left(\omega^{\prime} t+\pi-\varphi_{p} n\right)-\sin \left(\omega^{\prime} t-\varphi_{p} n\right)\right]= \\
=-\frac{l_{m} \tau}{\pi} B_{\delta m c}\left[\sin \left(\omega^{\prime} t+\pi-\frac{2 \pi p n}{z_{p}}\right)-\sin \left(\omega^{\prime} t-\frac{2 \pi p n}{z_{p}}\right)\right],
\end{gathered}
$$

where $\varphi_{p}$ - angle of current lag of a rotor;

$$
z_{p} \text { - number of rotor grooves. }
$$

Now it is possible to determine the electromotive force in the round equal to polar division:

$$
\begin{gathered}
-e=\frac{d \Psi}{d t}=-\frac{\omega^{\prime} l_{m} \tau w_{p}}{\pi} B_{\delta m c}\left[\cos \left(\omega^{\prime} t+\pi-\frac{2 \pi p n}{z_{p}}\right)-\right. \\
\left.-\cos \left(\omega^{\prime} t-\frac{2 \pi p n}{z_{p}}\right)\right]
\end{gathered}
$$

where

$$
z_{p} \text { - number of rotor grooves; }
$$

$$
\begin{aligned}
& w_{p} \text { - number of rounds of a phase of a rotor; } \\
& \Psi \text { - flux linkage. }
\end{aligned}
$$

Taking into account transformation of a formula (3), we will finally receive:

$$
e=\frac{2 \omega^{\prime} l_{m} \tau w_{p}}{\pi} B_{\delta m c} \cos \left(\omega^{\prime} t-\varphi_{p} n\right)
$$

We will define the magnetic flux falling on the first coil of a phase A:

$$
\begin{gathered}
\Phi_{1 k A}=l_{m} \int_{0}^{y \tau} B_{\delta m p}(x) d x=-l_{m} \int_{0}^{y \tau} B_{\delta m p} \sin \left(\omega t+\frac{\pi}{\tau} x-\varphi_{p e}\right) d x= \\
=\frac{l_{m} \tau}{\pi} B_{\delta m p}\left[\cos \left(\omega t+\pi y-\varphi_{p e}\right)-\cos \left(\omega t-\varphi_{p e}\right)\right],
\end{gathered}
$$

where $\quad \Phi_{1 k A}-$ magnetic flux falling on the first coil of a phase A;

$$
B_{\delta m p}-\text { maximum value of induction of the field of a }
$$

rotor;

$\varphi_{p e}-$ corner between the electromotive force and current of a rotor; stator;

$$
\omega-\text { angular frequency of rotation of the field of the }
$$

$$
y \text { - winding step relation to polar division. }
$$

We will define the stream falling on the second coil of a phase A:

$$
\begin{aligned}
\Phi_{2 k A}=l_{m} \int_{n_{2 A} \Delta x}^{y \tau+n_{2 A} \Delta x} B_{\delta m p}(x) d x=-l_{m} \int_{n_{2 A} \Delta x}^{y \tau+n_{2 A} \Delta x} B_{\delta m p} \sin \left(\omega t+\frac{\pi}{\tau} x-\varphi_{p e}\right) d x= \\
=\frac{l_{m} \tau}{\pi} B_{\delta m p}\left[\cos \left(\omega t+\pi y+\frac{\pi n_{2 A} \Delta x}{\tau}-\varphi_{p e}\right)-\right. \\
\left.-\cos \left(\omega t+\frac{\pi n_{2 A} \Delta x}{\tau}-\varphi_{p e}\right)\right]
\end{aligned}
$$

where $\Phi_{2 k A}-$ magnetic flux falling on the second coil of a phase A; second coil;

$n_{2 A}$ - number of tooth divisions of shift of the

$$
\Delta x \text { - length of one tooth division. }
$$

Knowing the value of a magnetic flux, we can determine the electromotive forces of the bobbin group of each phase. We will determine the electromotive force falling on the first coil of a phase A:

$e_{1 k A}=\frac{\omega}{\pi} l_{m} \tau B_{\delta m p}\left[\sin \left(\omega t+\pi y-\varphi_{p e}\right)-\sin \left(\omega t-\varphi_{p e}\right)\right] w_{k},(7)$

where $\quad w_{k}$ - number of rounds of one coil.

We will determine the electromotive force falling on the second coil of a phase A:

$$
\begin{gathered}
e_{2 k A}=\frac{\omega}{\pi} l_{m} \tau B_{\delta m p}\left[\sin \left(\omega t+\pi y+\frac{\pi}{\tau} n_{2 A} \Delta x-\varphi_{p e}\right)-\right. \\
\left.-\sin \left(\omega t+\frac{\pi}{\tau} n_{2 A} \Delta x-\varphi_{p e}\right)\right] w_{k}
\end{gathered}
$$

We will enter a concept of an angle of overlapping of the first and second coils. The size is universal and can consider the size of overlapping of various compass cards:

$$
\alpha_{o v}=\frac{\pi}{2}\left(y+\frac{n_{2 A} \Delta x}{\tau}\right),
$$

where second coils.

The final total expression for the electromotive forces of two coils, taking into account the angle of shift and the size of an angle of overlapping, takes a form:

$$
e_{1 k A}+e_{2 k A}=-2 \frac{\omega}{\pi} l_{m} \tau B_{\delta m p} w_{k}\left(1+\sin \alpha_{n e p}\right) \sin \left(\omega t-\varphi_{p e}\right) .
$$


Further we will define a full stream of one contour of a rotor of a component of the special electric drive. For this purpose it is necessary to know sizes of the magnetic fluxes connected with windings of the considered component and the electric drive in general. The design is arranged so that coils are switched on. Then we define the stream crossing the cross plane of the coil:

$$
\Phi_{\text {coil }}=2 \frac{2 i_{\hat{e}} w_{\hat{e}}}{R_{\mu \text { coil }}}=2 \Phi=\Phi_{m} \sin (\omega t),
$$

where $i_{\kappa}-$ instant current of the coil;

$R_{\mu c o i l}$ - magnetic resistance to a coil stream;

$\Phi_{\text {coil }}-$ magnetic flux of the coil.

We will consider the equations of an electric chain for a rotor. The fact that the system with two couples of poles and magnetic fluxes will be equal in each pole in size and a phase is important and alternate in the direction as all conductors currents are identical in size and a phase:

$$
\Phi_{p f}=\frac{4 i_{p} w_{p}}{R_{\mu k}}=\frac{4 I_{p} w_{p}}{R_{\mu k}} \cos \left(\omega^{\prime} t-\varphi_{p e}\right),
$$

where $\quad i_{p}-$ instant current of a rotor;

$$
\begin{aligned}
& R_{\mu k} \text { - magnetic resistance to a contour stream; } \\
& \Phi_{p f}-\text { magnetic flux of a contour. }
\end{aligned}
$$

Then the stream from all phases is equal to:

$$
\begin{gathered}
\Phi_{p \Sigma}=\frac{4 I_{p} w_{p}}{R_{\mu k}}\left[\cos \left(\omega^{\prime} t-\varphi_{p e}\right)+\frac{8,5}{9,5} \cos \left(\omega^{\prime} t-\varphi_{p e}-\frac{2 \pi}{19}\right)-\right. \\
-\frac{1}{9,5} \cos \left(\omega^{\prime} t-\varphi_{p e}-\frac{2 \pi}{19}\right)+\frac{7,5}{9,5} \cos \left(\omega^{\prime} t-\varphi_{p e}-\frac{4 \pi}{19}\right)- \\
-\frac{2}{9,5} \cos \left(\omega^{\prime} t-\varphi_{p e}-\frac{4 \pi}{19}\right)+\frac{6,5}{9,5} \cos \left(\omega^{\prime} t-\varphi_{p e}-\frac{6 \pi}{19}\right)- \\
-\frac{3}{9,5} \cos \left(\omega^{\prime} t-\varphi_{p e}-\frac{6 \pi}{19}\right)+\frac{5,5}{9,5} \cos \left(\omega^{\prime} t-\varphi_{p e}-\frac{8 \pi}{19}\right)- \\
-\frac{4}{9,5} \cos \left(\omega^{\prime} t-\varphi_{p e}-\frac{8 \pi}{19}\right)+\frac{4,5}{9,5} \cos \left(\omega^{\prime} t-\varphi_{p e}-\frac{10 \pi}{19}\right)- \\
-\frac{5}{9,5} \cos \left(\omega^{\prime} t-\varphi_{p e}-\frac{10 \pi}{19}\right)+\frac{3,5}{9,5} \cos \left(\omega^{\prime} t-\varphi_{p e}-\frac{12 \pi}{19}\right)- \\
-\frac{6}{9,5} \cos \left(\omega^{\prime} t-\varphi_{p e}-\frac{12 \pi}{19}\right)+\frac{2,5}{9,5} \cos \left(\omega^{\prime} t-\varphi_{p e}-\frac{14 \pi}{19}\right)- \\
-\frac{6}{9,5} \cos \left(\omega^{\prime} t-\varphi_{p e}-\frac{12 \pi}{19}\right)+\frac{2,5}{9,5} \cos \left(\omega^{\prime} t-\varphi_{p e}-\frac{14 \pi}{19}\right)- \\
-\frac{7}{9,5} \cos \left(\omega^{\prime} t-\varphi_{p e}-\frac{14 \pi}{19}\right)+\frac{1,5}{9,5} \cos \left(\omega^{\prime} t-\varphi_{p e}-\frac{16 \pi}{19}\right)-
\end{gathered}
$$

$$
\begin{gathered}
-\frac{8}{9,5} \cos \left(\omega^{\prime} t-\varphi_{p e}-\frac{16 \pi}{19}\right)+\frac{0,5}{9,5} \cos \left(\omega^{\prime} t-\varphi_{p e}-\frac{18 \pi}{19}\right)- \\
\left.-\frac{9}{9,5} \cos \left(\omega^{\prime} t-\varphi_{p e}-\frac{18 \pi}{19}\right)\right]
\end{gathered}
$$

where $\Phi_{p \Sigma}-$ total magnetic flux of a rotor.

Using trigonometrical transformations, we will finally receive:

$$
\Phi_{p \Sigma}=\frac{4 I_{p} w_{p}}{R_{\mu k}}\left[4 \frac{5}{14} \cos \left(\omega^{\prime} t-\varphi_{p e}\right)-\frac{1}{24} \sin \left(\omega^{\prime} t-\varphi_{p e}\right)\right]
$$

The formula (14) is expression for definition of the full magnetic flux connected with one contour of a squirrel cage of a rotor of a component of the special electric drive.

We will define a full magnetic flux of one bobbin group of a component of the special electric drive. For the chosen stator winding on forty eight grooves with two couples of poles [18], we will calculate the magnetic fluxes from all coils connected with the coil number of the one first bobbin group of phase A. Putting the received expressions, we will define a full stream of the first coil of the first bobbin group of phase A.

$$
\begin{aligned}
& \Sigma \Phi_{\text {coil } 1.1 A}=\Phi_{m}\left[\sin (\omega t)+\frac{7}{9} \sin (\omega t)+\frac{1}{9} \sin \left(\omega t+120^{\circ}\right)-\right. \\
& -\frac{5}{9} \sin \left(\omega t+120^{\circ}\right)-\frac{7}{9} \sin \left(\omega t+120^{\circ}\right)+\frac{1}{9} \sin \left(\omega t+240^{\circ}\right)+ \\
+ & \left.\frac{3}{9} \sin \left(\omega t+240^{\circ}\right)-\frac{5}{9} \sin \left(\omega t+240^{\circ}\right)-\frac{3}{9} \sin \left(\omega t+240^{\circ}\right)\right]= \\
= & \Phi_{m}\left[1 \frac{7}{9} \sin (\omega t)-1 \frac{2}{9} \sin \left(\omega t+120^{\circ}\right)-\frac{4}{9} \sin \left(\omega t+240^{\circ}\right)\right] .(15)
\end{aligned}
$$

Flux linkage of the considered coil is equal to:

$$
\begin{gathered}
\Psi_{\text {coil } 1.1 \mathrm{~A}}=\Phi_{m} w_{\text {coil }}\left[1 \frac{7}{9} \sin (\omega t)-1 \frac{2}{9} \sin \left(\omega t+120^{\circ}\right)-\right. \\
\left.-\frac{4}{9} \sin \left(\omega t+240^{\circ}\right)\right]
\end{gathered}
$$

We will define a magnetic flux of the second coil of the first bobbin group of phase A:

$$
\begin{aligned}
\Sigma \Phi_{\text {coil } 1.2 A}= & \Phi_{m}\left[1 \frac{7}{9} \sin (\omega t)-\frac{4}{9} \sin \left(\omega t+120^{\circ}\right)-\right. \\
& \left.-1 \frac{2}{9} \sin \left(\omega t+240^{\circ}\right)\right] .
\end{aligned}
$$

Flux linkage of the considered coil is equal to:

$$
\begin{gathered}
\Psi_{\text {coill.2A }}=\Phi_{m} w_{\text {coil }}\left[1 \frac{7}{9} \sin (\omega t)-\frac{4}{9} \sin \left(\omega t+120^{\circ}\right)-\right. \\
\left.-1 \frac{2}{9} \sin \left(\omega t+240^{\circ}\right)\right] .
\end{gathered}
$$


Total flux linkage of the first bobbin group of phase A is equal to:

$$
\begin{gathered}
\Psi_{\text {coil gð } 1 A}=\Phi_{m} w_{\text {coil }}\left[\frac{32}{9} \sin (\omega t)-\frac{15}{9} \sin \left(\omega t+120^{\circ}\right)-\right. \\
\left.-\frac{15}{9} \sin \left(\omega t+240^{\circ}\right)\right]
\end{gathered}
$$

where $\quad \Psi_{\text {coilgð } 1 A}-$ total flux linkage of the first bobbin group of phase A.

We will make trigonometrical transformations. As a result, the formula (19) takes a form:

$$
\begin{gathered}
\Psi_{\text {coil gð } 1 A}=\Phi_{m} w_{\text {coil }}\left\{\frac{32}{9} \sin (\omega t)-\frac{15}{9}[-\sin (\omega t)]\right\}= \\
=5 \frac{2}{9} \Phi_{m} w_{\text {coil }} \sin (\omega t),
\end{gathered}
$$

Flux linkage for the second coil will be analogous to a formula (20).

Knowing the expressions received on formulas (1) - (20), we can calculate the value of the reserved energy in the device. For this purpose we will define the expression for magnetic induction:

$$
B_{\Sigma}^{2}=\left(-B_{\delta m c} \cos \left(\omega+\frac{\pi}{\tau} x-\varphi_{c e}\right)-B_{\delta m p} \sin \left(\omega t+\frac{\pi}{\tau} x-\varphi_{p e}\right)\right)^{2},
$$

where $\quad B_{\delta m c}-$ maximum value of induction of the field of the stator;

$$
\varphi_{c e}-\text { corner between tension and current of the }
$$
stator.

Energy is determined by integration of a formula (21) by a gap surface:

$$
\begin{gathered}
W_{E M}=\frac{2 \delta l_{m}}{2 \mu_{0}}=\int_{0}^{2 \tau} A^{2} \cos ^{2}(\alpha)+ \\
+2 A B \cos (\alpha) \sin (\beta)+B^{2} \sin ^{2}(\beta),
\end{gathered}
$$$$
\text { where } \quad A=-B_{\delta m c} \text {; }
$$

$$
\begin{aligned}
& B=-B_{\delta m p} \\
& \alpha=\omega t+\frac{\pi}{\tau} x-\varphi_{c e} ; \\
& \beta=\omega t+\frac{\pi}{\tau} x-\varphi_{p e}
\end{aligned}
$$

Finally the formula (22) accepts the value:

$$
W_{E M}=\frac{\delta l_{m} \tau}{\mu_{0}}\left(B_{\delta m c}^{2}+B_{\delta m p}^{2}-2 B_{\delta m c} B_{\delta m p} \sin \left(\varphi_{p e}-\varphi_{c e}\right)\right)
$$

The conducted research of electrical power processes in special electric drives with asynchronous engines makes it possible to define force of instant effort and the moment on a shaft.

For this purpose the volume of the system is presented in the form of characteristic elementary volumes. In each volume energy is determined by a formula (23). All elementary values are summarized and total electromagnetic energy of all device of time and for this spatial arrangement of a rotor, concerning the stator, is defined at present.

As distribution of induction in a gap is based by means of the developed programs of calculation, there is no analytical expression of this dependence for definition of effort use numerical differentiation of private derivative energy $W_{x}$ on movement coordinate on a surface of a rotor $x$. Considering that the dependence $W(s)$ is rather smooth (Fig.1), the threepoint template giving the good accuracy of numerical differentiation is applied in finding of a derivative.

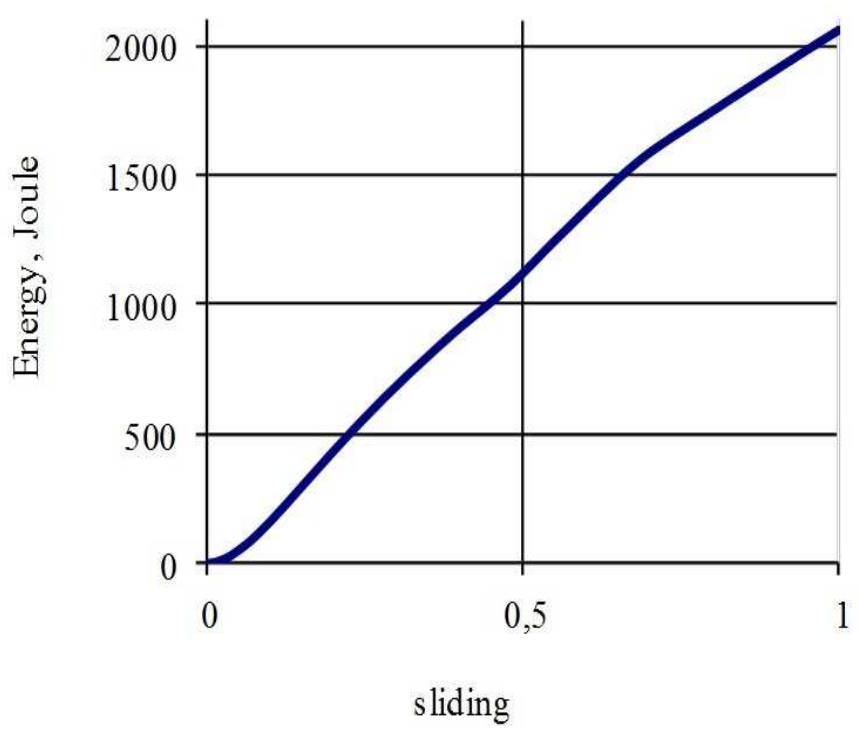

Fig. 1. Schedule of dependence of energy on sliding.

We will consider points $x+\Delta x, \mathrm{x}$ and $x-\Delta x$. At the same time $\Delta x$, it is possible to take a rather small size. We consider any time point when axes of fields of the stator and a rotor are located with a shift reletively each other. We find provisions of axes, total distribution $B(x)$, and the reserved energy $W_{x}$, displacing an axis of the field of a rotor in a point $-\Delta x$ that corresponds to lag of a rotor and an increase in sliding of $s$. It follows from the fact that the field moves from right to left. The rotor moves in the same direction. The point $-\Delta x$ will correspond to lag of a rotor. For a point $-\Delta x$ we define total distribution $B(x)$ and the corresponding reserved energy $W_{x-\Delta x}$. Displacing an axis of the field of a rotor in a point $+\Delta x$ that corresponds to advancing of a rotor and a reduction of sliding, we repeat the same calculations and we find the corresponding 
energy $W_{x+\Delta x}$. Results of calculation are shown in Fig.2 and Fig.3.

Calculations can be made for any time point, i.e. for any angle of rotation of a rotor concerning the stator. By means of the developed programs of calculation, we receive an instant value of force and the moment operating on a shaft of the electric drive for a whole revolution of a shaft.

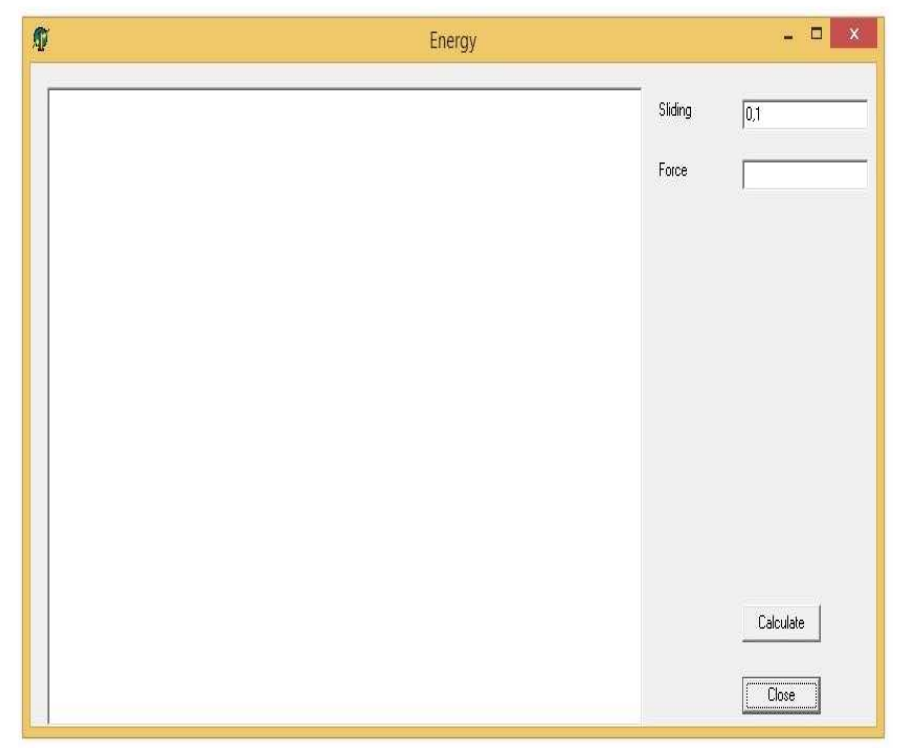

Fig. 2. Data input.

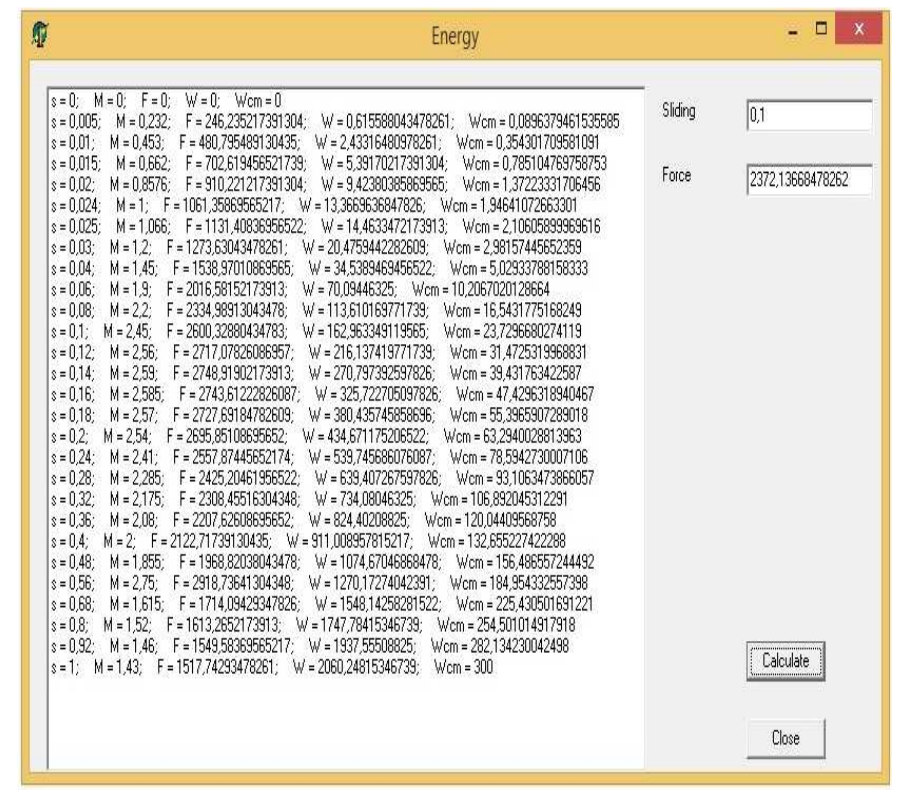

Fig. 3. Obtaining result of calculations.

\section{CONCLUSION}

The research of electrical power processes for optimum modeling and design of special electric drives with asynchronous engines was conducted. Results were received, allowed one to carry out mathematical modeling of the electromechanical processes happening in the electric drive and their components. Interdependence in space and in time of magnetic fluxes, tension, electric currents, the electromotive forces of a self-induction and interinduction in stator and rotor windings components of the studied devices were received. It in turn allowed defining the electromagnetic energy reserved in the device. The carried-out analysis showed that the electrical power and electromechanical parameters calculated by means of the developed programs were received with high precision and coincided with a control example and with passport data of the existing devices. It allowed using new theoretical approaches to design and modeling of the developed special systems of the asynchronous electric drive and its components.

\section{References}

[1] A.V. Egorov, A.N. Komkov, G.N. Malinovskaya, On the issue of interaction of electric drives as a part of the electrical system, Territory neftegaz, Vol. 2. pp. 106-112, 2016.

[2] A. E. Kozyaruk, Energy efficient electromechanical systems of mining and transport machines, Notes of mining institute, vol. 218. pp. 261-269, 2016.

[3] N. S. Lot, O. I. Osipov, A. M. Zhidkov, Future prospects of winder electric drives, Drives and components of machines, Vol. 6 (22). pp. 912, 2016.

[4] I. P. Kopylov, Design of electrical machines, M. High. sch., pp. 757, 2002.

[5] Stumberger Bojan, Stumberger Gorazd, Hadciselimoviz Miralem, Zagradisnik Ivan. Torque ripple reduction in exterior-rotor permanent magnet synchronous motor, J. Magn. and Magn. Mater, Vol. 2, pp. E826-E828, 2006.

[6] A.B. Petrochenkov, Performance functions for basic electrical equipment of the mineral resources industry, Russian Electrical Engineering, Vol. 11. pp. 634-639, 2015.

[7] A. A. Ali Salama, N. K. Andreev, Study of effectiveness of pi and neural network speed controllers in indirect vector control of induction motor using stator flux model, News of higher educational institutions. Power problems, Vol. 3-4, pp. 101-108, 2012.

[8] A. E. Kozyaruk, B. Yu. Vasiliev, Methods and tools increasing energy efficiency of machines and technologies with asynchronous drives, Bulletin of the Southern Ural State University, Series: Power, vol. 15, № 1, pp. 47-53, 2015.

[9] O.I. Osipov, Problems of implementation and adjustment of the modern electric drives, Russian Electrical Engineering, vol. 86, № 1, pp. 5-8, 2015.

[10] D. M. Isaev, A. A. Borovik, O. I. Osipov, Technological Requirements For Electric Drive Pulling Device Of Horizontal Continuous Casting Machine, Drives and components of machine, Vol. 9(5-6), pp. 2-5, 2013.

[11] V.U. Kornilov, A.N. Tsvetkov, A.I. Muchametshin, Investigation of the process of electromagnetic energy conversion in an asynchronous motor with a combined two-layer winding, Nonlinear world, № 6, pp. 33-39, 2017.

[12] K. N. Bayramkulov, V. I. Astakhov, Calculation of magnetic field in the environment with non-uniform and anisotropic properties on the basis of the electric chain of Kirchhoff, News of higher educational institutions. Electromechanics, Vol. 1, pp. 3-11, 2010.

[13] N. Schmitz, D. Novotny, Introduction to Electromechanics, M. Energy, pp. 336, 1969.

[14] R. T. Akhmetgarayev, N. K. Andreev, Direct torque control in the alternated current induction motors of sucker rod pumps in oil production, News of higher educational institutions, Power problems, Vol. 9-10, pp. 100-104, 2011.

[15] V. Yu. Karandey, B. K. Popov, O. B. Popova, V. L. Afanasyev, Determination of power and moment on shaft of special asynchronous electric drives, IOP Conf. Series: Materials Science and Engineering, Vol. 327, 052003, 2018. doi:10.1088/1757-899X/327/5/052003.

[16] V. Yu. Karandey, B. K. Popov, O. B. Popova, V. L. Afanasyev, Optimization of parameters of special asynchronous electric drives, IOP 
Conf. Series: Materials Science and Engineering, Vol. 327, 052002, 2018. doi:10.1088/1757-899X/327/5/052002.

[17] A.S. Anuchin, Yu. M. Hanova, I.V.Gulyaev, Development of the method of fast and exact modelling of electric drives, Industrial power, Vol. 4, pp. 28-33, 2016.
[18] M. P. Kostenko, L.M. Piotrovsky, Electrical machines, L. Energy, 1973. 